\title{
Characterization and Analysis of Triaxially Braided Polymer Composites under Static and Impact Loads
}

\author{
Robert K. Goldberg ${ }^{1}$, Gary D. Roberts ${ }^{2}$, Brina J. Blinzler ${ }^{3}$, Lee W. Kohlman ${ }^{4}$ and \\ Wieslaw K. Binienda ${ }^{5}$ \\ ${ }^{1}$ NASA Glenn Research Center, Mechanics and Life Prediction Branch, Cleveland, \\ OH 44135; email: Robert.K.Goldberg@nasa.gov \\ ${ }^{2}$ NASA Glenn Research Center, Polymers Branch, Cleveland, OH 44135; email: \\ Gary.D.Roberts@nasa.gov \\ ${ }^{3}$ University of Akron, Civil Engineering Department, Akron, OH 44325; email: \\ bjc43@zips.uakron.edu \\ ${ }^{4}$ University of Akron, Civil Engineering Department, Akron, OH 44325; email: \\ lwk1@zips.uakron.edu \\ ${ }^{5}$ University of Akron, Civil Engineering Department, Akron, OH 44325; email: \\ wieslaw@uakron.edu
}

\begin{abstract}
In order to design impact resistant aerospace components made of triaxiallybraided polymer matrix composite materials, a need exists to have reliable impact simulation methods and a detailed understanding of the material behavior. Traditional test methods and specimen designs have yielded unrealistic material property data due to material mechanisms such as edge damage. To overcome these deficiencies, various alternative testing geometries such as notched flat coupons have been examined to alleviate difficulties observed with standard test methods. The results from the coupon level tests have been used to characterize and validate a macro level finite element-based model which can be used to simulate the mechanical and impact response of the braided composites. In the analytical model, the triaxial braid unit cell is approximated by using four parallel laminated composites, each with a different fiber layup, which roughly simulates the braid architecture. In the analysis, each of these laminated composites is modeled as a shell element. Currently, each shell element is considered to be a smeared homogeneous material. Simplified micromechanics techniques and lamination theory are used to determine the equivalent stiffness properties of each shell element, and results from the coupon level tests on the braided composite are used to back out the strength properties of each shell element. Recent improvements to the model include the incorporation of strain rate effects into the model. Simulations of ballistic impact tests have been carried out to investigate and verify the analysis approach.
\end{abstract}

\section{INTRODUCTION}

The use of textile (woven and braided) composites is being actively investigated for application in aerospace structures, as an alternative to metals or traditional laminated composites. For example, triaxially-braided composites are being utilized in fan 
cases for jet engines. To be certified as flight worthy, the fan case must demonstrate the capability to contain a released fan blade and maintain structural integrity. As part of the design process the need exists to simulate the case deformation, damage and failure during a blade out event through the use of transient dynamic finite element codes. The reliability of the impact simulations for aircraft components made with triaxially-braided carbon fiber composites is currently limited by inadequate material property data and a lack of validated material models for analysis.

In order to properly characterize and validate the material models required for an impact simulation, as well as to understand the key material mechanisms which need to be simulated by a material model, appropriate experimental data is required. Littell, et al (2009a) conducted an extensive series of coupon tests, utilizing standard straight-sided coupons, on representative triaxially braided composites. Through the use of digital image correlation (DIC) methods, Littell, et al correlated key features of the macroscopic stress strain curve to matrix microcracking events within fiber tows (hereafter referred to as "fiber splitting") and local out-of-plane delaminations. Another key finding made by Littell, et al was that during the transverse tensile tests, a significant amount of edge damage, resulting in large regions of low strain and premature failure (reflected in artificially low failure stresses), took place. The edge damage also resulted in regions of artificially low stress, which resulted in the stressstrain curves obtained during the transverse tensile tests most likely not being representative of the large scale composite response and therefore of limited use in the characterization of material models. These results identified the need to develop modified test methods to obtain improved transverse tensile data that would be more appropriate to use in model characterization.

Significant amounts of research has been conducted in the analysis and modeling of textile composites. Representative examples include work conducted by Chou and Ishikawa (1989), Byun (2000), Tanov and Tabiei (2001) and Bednarcyk and Arnold (2003). In each of these methods, analytical techniques have been utilized to compute the effective properties of the composite, and these homogenized properties can then be utilized within a finite element analysis to generate the effective response of the material. However, for triaxially-braided composites, Roberts et al (2002) found that when these materials are subject to impact, oftentimes the damage will propagate along the fiber directions. To best simulate these damage patterns, the braid architecture should be directly simulated within the finite element model. To account for the fiber architecture in a computationally efficient manner, Cheng (2006), Littell, et al (2009b), and Goldberg, et al (2010) created a "Braided Through the Thickness” approach, where the braided composite unit cell is modeled as a series of parallel shell elements, which each element is a laminated composite with the appropriate fiber layup. The model was developed within the context of LS-DYNA (LSTC, 2007), a commercially available transient dynamic finite element code which is commonly used within the aerospace industry. A continuum damage mechanicsbased composite constitutive model available within LS-DYNA was used as the material model. 
In the current paper, alternative test methods that have been developed to obtain improved transverse tension results will be described. The methodology that has been developed to simulate the response of textile composites under impact conditions, including the architecturally-dependent damage, will be presented. The methods in which the experimental data is used to characterize the required input parameters for the analysis approach will also be discussed. Recent efforts to incorporate the effects of strain rate into the impact simulations will be described.

\section{EXPERIMENTAL METHODS AND RESULTS}

A series of composite panels were fabricated by resin transfer molding using twodimensional triaxial-braided preforms with a $\left[0 \%+60^{\circ} /-60^{\circ}\right]$ architecture and $177^{\circ} \mathrm{C}$ cure epoxy resins. Six layers of the braided plies were stacked to make the complete test specimen. A high strength, standard modulus carbon fiber, TORAYCA T700S (Toray Carbon Fibers America, Inc.), was used for all of the panels. Several matrix materials were used. For this paper, results will be presented on studies using the matrix materials EPIKOTE Resin 862/EPIKURE Curing Agent W (Hexion Specialty Chemicals) (E-862 for short), and CYCOM PR 520 matrix (Cytec Industries, Inc.) (PR 520 for short), a one-part toughened resin. The braid preform had $24 \mathrm{k}$ tows in the $0^{\circ}$ (axial) direction and $12 \mathrm{k}$ tows in the $\pm 60^{\circ}$ (bias) directions. The fiber bundle spacing in the axial and bias directions were adjusted to give the same fiber volume fraction in the axial and bias directions. The fiber architecture was designed to yield a quasi-isotropic stiffness response. The fiber volume fraction in the fabricated panels was 0.56 .

A series of tensile tests were performed using an axial/torsion test machine. All of the coupon tests were conducted under displacement control at a rate of $1.27 \mathrm{~mm} / \mathrm{min}$ in accordance with ASTM D3039 Section 11.3.2. Two flat coupon geometries were used for the tensile testing: a straight-sided coupon based on ASTM D3039 and a double edge notch coupon. Both coupon geometries are shown in Figure 1. Both geometries were used for axial and transverse tension tests, where for the axial tests the $0^{\circ}$ fibers were parallel to the load direction, and for the transverse tests the $0^{\circ}$ fibers were transverse to the load direction. In the transverse straight-sided coupon tests, no tows extended from grip to grip, which would allow edge initiated damage to propagate along the unconstrained fiber tow. In the axial straight-sided coupon tests, and both axial and transverse notched coupon tests, at least some tows were gripped end to end, which allowed the load to be carried beyond the stress which initiates edge damage. A commercially-available digital image correlation (DIC) measurement system (GOM: ARAMIS) was used to obtain full-field surface strain measurements. The DIC measurements can also be used to provide information on local surface damage, as discussed by Littell, et al (2009a).

As discussed by Littell, et al (2009a), edge damage was identified as a significant issue during straight-sided transverse tensile tests, and a probable key contributing factor to the artificially low transverse tensile strength measurements made during these tests. Figure 2 shows edge damage in a transverse tensile test before failure and 
the resulting shear type failure observed after the test. In this figure, the failure initiated as damage on the right edge and propagated as a shear failure along the $+60^{\circ}$ and $-60^{\circ}$ fiber directions.

To alleviate the edge damage and failure, the notched coupon geometry shown in Figure 1, mentioned above, was tested in transverse tension. As shown in Table 1, the transverse tension strength values obtained using the notched coupon geometry were significantly higher than the strength values obtained using the straight-sided coupon tests for both materials. While intuitively one would think that the strength of a notched coupon would be lower than the strength of an unnotched coupon, the notched coupons had much smaller areas of edge damage with low stresses, leading to higher overall strength values. While not shown in the table, the axial tensile strengths measured using the notched coupon geometries were quite close to the transverse tension strengths, which is much more representative of the expected material response. However, the measured strengths may still not be totally indicative of the actual material response as the notched specimens displayed stress concentrations at the notch tips and yielded a biaxial stress response. Testing of tubes, in which edge damage will not be an issue and artificial stress concentrations should not be present is currently underway which should provide insight into the accuracy of the results obtained using the notched coupon tests.

\section{ANALYTICAL METHODOLOGY}

In the analytical model as developed by Cheng (2006), Littell, et al (2009b), and Goldberg, et al (2010), the braided composite is discretized into a series of parallel shell elements, each of which is modeled as a laminated composite. To discretize the composite unit cell, as shown in Figure 3(a) and 3(b) the composite unit cell is subdivided into four subcells, labeled “A”, "B", “C” and "D". Subcells A and C consist of both axial and bias fibers, while subcells B and D consist of bias fibers only and matrix pockets. As shown in Figure 3(c), each subcell is modeled as a laminated composite. Each subcell is assumed to have equal thickness. The fact that subcells A and C have more fiber layers than subcells $B$ and $D$ is reflected in the fact that subcells $\mathrm{A}$ and $\mathrm{C}$ have a higher local fiber volume fraction than subcells $\mathrm{B}$ and $\mathrm{D}$. The overall composite is modeled by stacking six unit cells on top of each other. In the finite element analysis, each subcell is modeled as an independent shell element, with smeared, orthotropic stiffness and strength properties representative of the laminated composite simulated by the subcell composite layup shown in Figure 3(c).

The commercial transient dynamic finite element code LS-DYNA (LSTC, 2007) was utilized for analyses described in this paper. The composite material model *MAT_58, otherwise known as *MAT_LAMINATED_COMPOSITE_FABRIC, available within LS-DYNA was utilized as the constitutive model for the analyses. *MAT_58 is a continuum damage mechanics model based on a theory developed by Matzenmiller et al (1995) in which all of the nonlinearity present in the composite response is assumed to be due to damage mechanisms. To utilize the material model, the smeared, orthortropic material axis system stiffness and strength values in all of 
the coordinate directions needed to be input for each subcell. Note that due to the differing fiber layup and fiber volume fractions, one set of material properties was required for subcells $\mathrm{A}$ and $\mathrm{C}$, and another set of properties was required for subcells $\mathrm{B}$ and $\mathrm{D}$.

For the composite materials examined in this study, equivalent unidirectional laminates could not be made and tested, which resulted in indirect methods being needed to obtain the required stiffness and strength properties for each subcell. Details of the methodology utilized to obtain the needed properties are described in Goldberg, et al (2010), with only a brief summary being provided here. To obtain the equivalent stiffness properties for each subcell, constituent level (fiber and matrix) elastic properties were utilized within a micromechanics approach, and then combined with composite laminate theory to compute the needed values. To obtain the required strength values, mechanics of materials theory is used in combination with global coupon stress-strain curves in the various directions (axial tension, transverse tension, axial compression, transverse compression and in-plane shear) to back out the required strength values for each subcell. The baseline values are computed based on quasi-static test results, and as will be described later in this paper selected results obtained at higher strain rates are used to quantify strain rate effects in the material response. By utilizing this type of methodology, only global coupon level experimental data for the braided composite is required, which is relatively easy to obtain. Furthermore, any in-situ effects such as material aging and weak interfaces are implicitly accounted for. By utilizing coupon level testing to characterize the model, the impact analyses become predictive instead of correlative. However, the material characterization methodology underscores the need for accurate coupon level test data. For example, without reliable transverse tension strength values, approximations must be made in the material characterization process.

As discussed by Littell, et al (2009b), the actual material response of the types of composites examined in this study is most likely strain rate dependent. Therefore, a need exists to incorporate strain rate dependence into the modeling methodology. Some preliminary analytical studies indicated that the local strain rates may reach a value as high as 2400 /s. To include strain rate effects into the analysis, the LSDYNA material model *MAT_158, or *Mat_Rate_Sensitive_Composite_Fabric, was employed. *MAT_158 is an extension of the *MAT_58 material which incorporates strain rate effects by using a viscoelasticity approach through applying a set of Prony series. The same material parameters as utilized within the original *MAT_58 are used, with the addition of an effective shear modulus and a decay constant. The scale factors are assumed to be identical in all of the material directions. For this study, the shear modulus for each subcell was computed using the axial modulus and Poisson's ratio. The decay constant was defined as the longitudinal modulus for each subcell divided by the applied strain rate multiplied by the axial tensile strength at the defined strain rate. Due to the fact that subcells $A$ and $C$ had a large volume fraction of carbon fibers, which can reasonably be assumed to be strain rate independent, the material response of these subcells were assumed to be independent of strain rate. Since subcells B and D had a much lower fiber volume fraction, the response of these 
subcells was assumed to vary with strain rate, and the material parameters were adjusted accordingly.

\section{ANALAYTICAL RESULTS}

The baseline quasi-static material properties for both the T700/E-862 and T700/PR 520 materials were determined using the procedures described in this paper and are listed in Goldberg, et al (2010). The effects of strain rate were incorporated into the analysis of the T700/PR 520 material. Limited high strain rate experimental data obtained by Pereira et al (2010) was utilized in combination with strain rate dependent stress-strain curves obtained analytically by Liu (2011) to characterize the strain rate-dependent response of the material. The shear modulus was computed using the data shown in Goldberg et al (2010). The decay constant for each strain rate was computed using the procedure described above and the values are listed in Table 2.

Impact analyses were conducted to simulate the conditions of impact experiments conducted by Pereira et al (2010). The composite panels were $0.3048 \mathrm{~m}$ square by $0.0032 \mathrm{~m}$ thick. The panels were held in a circular fixture with an aperture of 0.2054 m. Thin walled aluminum 2024 projectiles with properties and dimensions specified by Pereira et al (2010) were used. To model the impact tests, the flat panels were modeled using 2700 shell elements as is shown in Figure 4. The circular fixture restraint was simulated by fully constraining all of the nodes along the circular boundary. The projectile was modeled as a linear elastic material.

Experimental and simulated results from impact tests conducted on the T700/E-862 material are shown in Figure 5. As can be seen in the figure, the simulated ballistic limit of $160 \mathrm{~m} / \mathrm{s}$ is only slightly below the experimentally-determined ballistic limit of $166 \mathrm{~m} / \mathrm{s}$. The simulation does capture the progression of damage along the features of the braid architecture. The experimental damage patterns are somewhat more elongated than the simulated damage patterns. Overall, the simulation appears to reasonably capture the features of the impact result. The hole produced by the projectile passing through the test specimen is more pronounced in the simulations than was observed in the actual experiments. This result is due to the fact that in the actual test specimen continuous off-axis fibers are present which can "snap back" after the projectile passes though. Since the method used to approximate the braid architecture in the simulations does not include continuous fibers, this phenomenon cannot be simulated. As will be discussed in more detail momentarily, some of the discrepancy between the experimental and simulated results could be due to only quasi-static properties being used in the simulation, and by properly including the strain rate dependent response of the material improved predictions could be obtained.

To demonstrate this concept of the effects of strain rate on the simulated impact response on the material, simulations of the impact response of the T700/PR 520 material were conducted and the results are shown in Figure 6. Two sets of simulations were conducted; one in which only quasi-static properties were used and 
one in which strain rate dependent material properties were used. No experimental impact data was available for this material, so only qualitative observations can be made. As can be seen in Figure 7, utilizing the strain rate-dependent material properties resulted in a relatively slight increase in the predicted ballistic limit, from $221 \mathrm{~m} / \mathrm{s}$ to $236 \mathrm{~m} / \mathrm{s}$. However, the predicted impact damage patterns were quite different between the two simulations. These results indicate that properly accounting for the strain rate dependent response of the material could be significant in properly simulating the impact response of the braided composites.

\section{CONCLUSIONS}

A combined experimental and analytical study has been conducted to more completely understand the material mechanisms related to the mechanical response of triaxially-braided polymer matrix composites, and to develop the ability to accurately simulate the mechanical and impact response of these materials. Improved coupon test methods have been developed to obtain more accurate values of key mechanical properties, such as the transverse tensile strength. A macroscopic finite elementbased method has been developed to simulate the impact response of the braided composites, including the details of the architecturally-based damage patterns. The method has been modified to allow for the incorporation of the strain rate dependence of the material response into the impact simulations. The analytical methods have displayed promise in providing the ability to accurately predict the ballistic limit and impact damage patterns of braided composites. Future work will include the development of tube test methods to more completely characterize the material behavior and modification of the analysis approach to more accurately represent the impact damage patterns of braided composites.

\section{REFERENCES}

Bednarcyk, B.A., and Arnold, S.M. (2003). "Micromechanics-Based Modeling of Woven Polymer Matrix Composites.” AIAA Journal, 41(9), 1788-1796.

Byun, J.-H. (2000). "The analytical characterization of 2-D briaded textile composites.” Composites Science and Technology, 60(5), 705-716.

Cheng, J. (2006). "Material Modeling of Strain Rate Dependent Polymer and 2d Triaxially Braided Composites.” Ph.D. Dissertation, University of Akron.

Chou, T.W., and Ishikawa, T. (1989). "Analysis and Modeling of TwoDimensional Fabric Composites.” Textile Structural Composites, Elseveir Schence Publishers, 209-277.

Goldberg, R.K. et al (2010). "Modification of a Macromechanical Finite-Element Based Model for Impact Analysis of Triaxially-Braided Composites.” NASA TM2010-216922.

Liu, K.C. (2011). "Micromechanics Based Multiscale Modeling of the Inelastic Response and Failure of Complex Architecture Composites.” Ph.D. Dissertation, Arizona State University.

Littell, J.D. et al (2009a). "Characterization of Damage in Triaxial Braided Composites under Tensile Loading.” Journal of Aerospace Engineering, 22(3), 270279. 
Littell, J.D. et al (2009b). "Effect of microscopic damage events on static and ballistic impact strength of triaxial braid composites.” Composites: Part A, 40, 18461862.

LSTC (2007). LS-DYNA Keyword Manual v. 971. Livermore, CA.

Matzenmiller, A. et al (1995). “A Constitutive Model for Anisotropic Damage in Fiber-Composites.” Mechanics of Materials, 20, 125-152.

Pereira, J.M. et al (2010). "Experimental Techniques for Evaluating the Effects of Aging on Impact and High Strain Rate Properties of Triaxial Braided Composite Materials.” NASA TM-2010-216763.

Roberts, G.D. et al (2002). "Impact Testing and Analysis of Composites for Aircraft Engine Fan Cases.” NASA TM-2002-211493.

Tanov, R., and Tabiei, A. (2001). "Computationally Efficient Micromechanical Models for Woven Fabric Composite Elastic Moduli.” Journal of Applied Mechanics, 68(4), 553-560.

Table 1. Comparison of Transverse Tension Strength of Straight-Sided and Notched Specimens

\begin{tabular}{|c|c|c|}
\hline $\begin{array}{c}\text { Transverse Tensile } \\
\text { Strength (MPa) }\end{array}$ & T700/E-862 & T700/PR 520 \\
\hline Straight-Sided & 462.0 & 559.8 \\
\hline Notch & 813.6 & 722.3 \\
\hline
\end{tabular}

Table 2. Decay Constant Values for T700/PR 520 Material.

\begin{tabular}{|c|c|}
\hline Strain Rate (1/s) & Decay Constant \\
\hline 420 & $2.26 \times 10^{4}$ \\
\hline 1500 & $8.10 \times 10^{4}$ \\
\hline 2500 & $1.36 \times 10^{5}$ \\
\hline
\end{tabular}

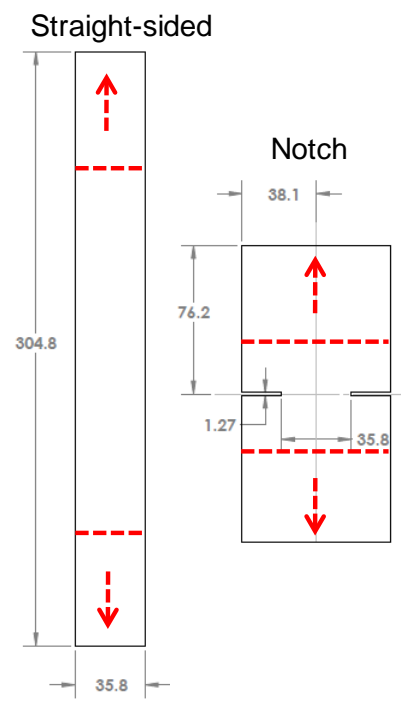

Figure 1. Straight-sided and notched coupon geometry. 

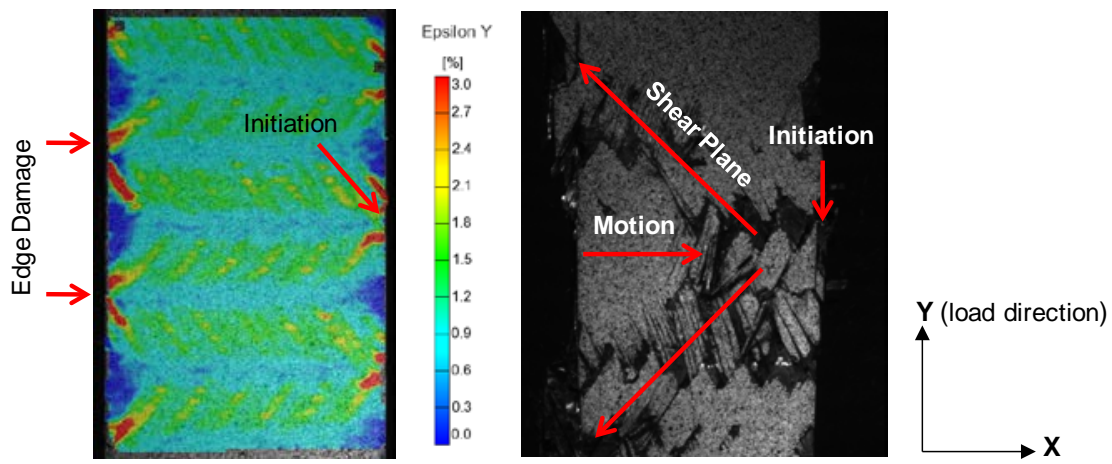

Figure 2. Edge damage in a transverse tension specimen identified by DIC (left and shear failure (right).

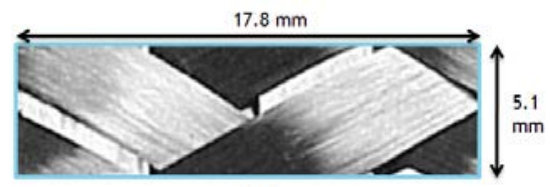

(a)
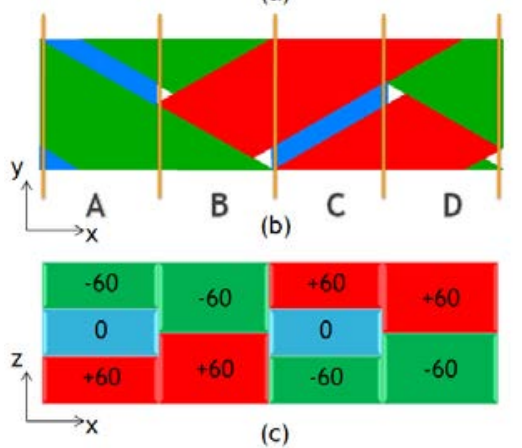

Figure 3. Discretization of unit cell a.) Digital image of unit cell. (b) Identification of axial and bias fibers in unit cell. (c) Approximation of braid architecture of unit cell.

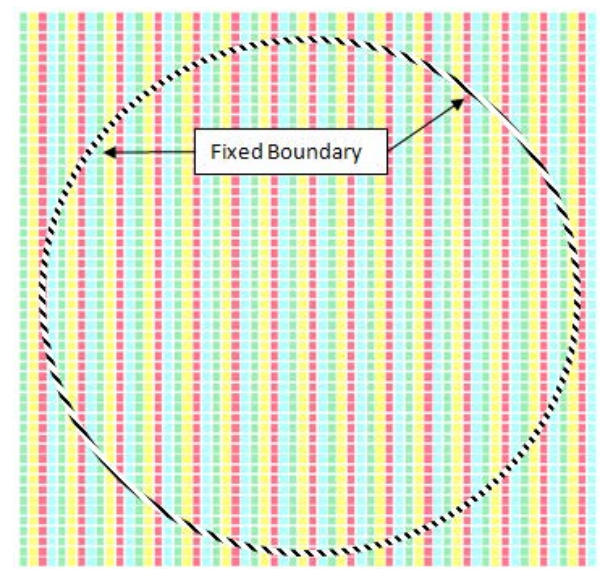

Figure 4. Finite Element Mesh for Impact Simulations. 


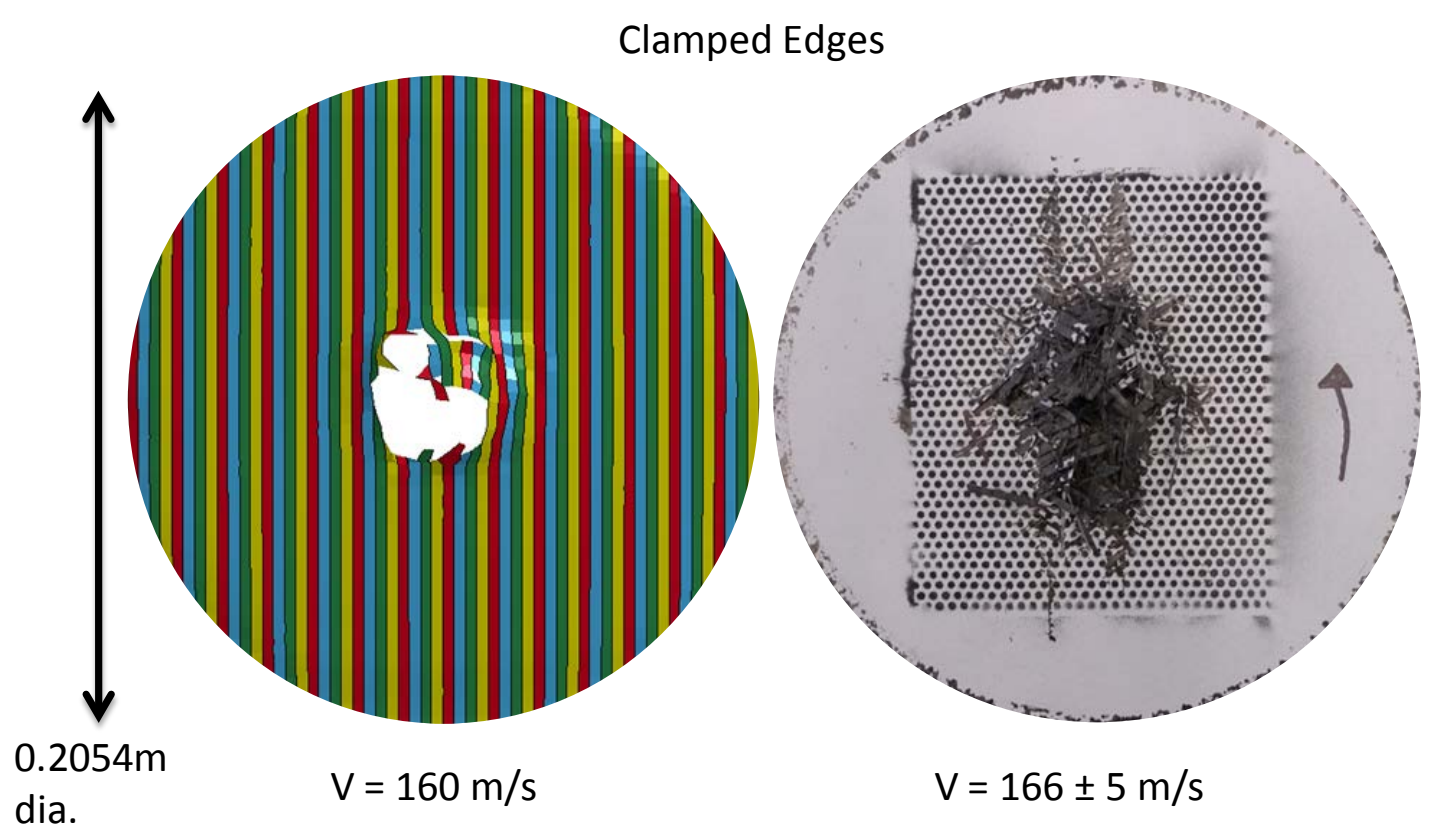

Figure 5. Simulated (left) and experimental (right) damage patterns for T700/E862 composite subject to impact.

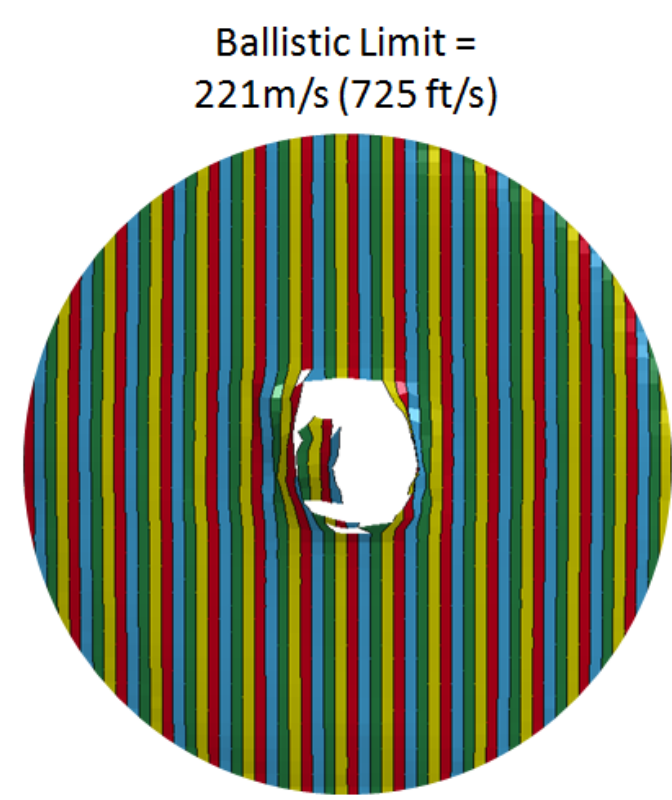

Static Properties

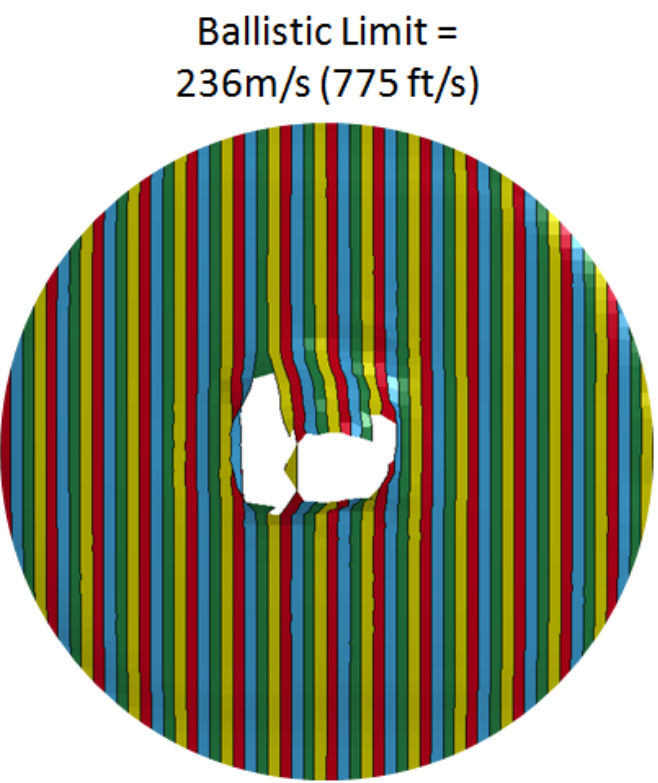

Strain Rate Properties

Figure 6. Comparison of impact simulations of T700/PR 520 material using quasi-static (left) and strain rate dependent (right) material properties. 